\title{
Asymptotic Degree Distribution of a Kind of Asymmetric Evolving Network
}

\author{
Zhimin Li, ${ }^{1}$ Zhaolin $\mathrm{He}^{2}$ and Chunhua $\mathrm{Hu}^{3}$ \\ ${ }^{1}$ School of Mathematics and Physics, Anhui Polytechnic University, Wuhu, Anhui 241000, China \\ ${ }^{2}$ School of Management Engineering, Anhui Polytechnic University, Wuhu, Anhui 241000, China \\ ${ }^{3}$ School of Applied Mathematics, Beijing Normal University Zhuhai, Zhuhai, Guangdong 519087, China
}

Correspondence should be addressed to Zhimin Li; zmli08@ahpu.edu.cn

Received 19 May 2014; Accepted 4 June 2014; Published 9 July 2014

Academic Editor: Hongli Dong

Copyright (C) 2014 Zhimin Li et al. This is an open access article distributed under the Creative Commons Attribution License, which permits unrestricted use, distribution, and reproduction in any medium, provided the original work is properly cited.

We propose a kind of evolving network which shows tree structure. The model is a combination of preferential attachment model and uniform model. We show that the proportional degree sequence $\left\{p_{k}\right\}_{k>1}$ obeys power law, exponential distribution, and other forms according to the relation of $k$ and parameter $m$.

\section{Introduction}

In recent ten years, there has been much interest in understanding the properties of real large-scale complex networks which describe a wide range of systems in nature and society. Examples of such networks appear in communications, biology, social science, economics, and so forth [1]. In pursuit of such understanding, mathematicians and physicists usually use random graphs to model all these real-life networks. In the investigation of various complex networks, the degree distribution is always the main concern because it characterizes the fundamental topological properties of complex networks which show importance in network control, estimation, and sensor [2-8]. Several models were introduced to explain the properties. Bollobás [9] proposed a model with $n$ vertices and $m$ edges. In this model, the degree distribution is approximately Poisson distribution. Later, Barabási and Albert [10] proposed the following model: at each time step, add a new vertex $v$ and a fixed number $r$ of edges originating at $v$ and directed towards vertices chosen at random with probability proportional to their degrees. Based on simulation and heuristic approximation, they predicted that the degree distribution behaves like $d^{-3}$ for all $r \geq 1$. The result was confirmed by Barabási et al. [11, 12 ]. In order to generate power laws with arbitrary exponents,
Dorogovtsev et al. [13] and Drine et al. [14] introduced the following natural generalization of the above model: the destination of the $r$ new edges added at each time step is chosen with probability proportional to the degree plus an initial attractiveness $\alpha r$; they gave a nonrigorous argument that the degree distribution $p_{d}$ behaves like $d^{-2-\alpha}$ for large $d$.

In some real networks, experiments show that the distribution obeys neither power law nor exponential. To explain the phenomenon, we propose a model as follows: starting with a single vertex, at each time step, a new vertex is added and linked to one of the existing vertices, which is chosen according the following rule: at time $m, 2 m, 3 m, \ldots$, where $m$ is integer, we choose one of the existing vertices with probability proportional to the degree; that is, we have probability $k / s_{n}$, where $k$ is the degree of the vertex chosen and $s_{n}$ is the total degree of vertices; at another time step, we choose one of the existing vertices with equal probability. Related models were also proposed by Krapivsky and Redner [15] and $\mathrm{Li}[16]$ to describe the organization of growing networks. In this paper, we will focus on the distribution of evolving network and the distribution of the number of vertices with given degree will be considered in Section 2 . In Section 3, we will consider the asymptotic degree distribution. 


\section{The Number of Vertices with Given Degrees}

Let $D_{n}(k)$ denote the number of vertices with degree $k$ at time $n$. We will consider the case $k=1,2$ in this section and the case $k>2$ will be considered in the next section. As $k=1$, we obtain the following result.

Lemma 1. In the evolving network, the expectation of the number of degree 1 satisfies

$$
E D_{n}(1)=\frac{2 m}{4 m-1} n \text {. }
$$

Proof. Herein after, $=$ denotes asymptotic equivalence as $n \rightarrow$ $\infty$. From the way the network is formed, we can see that, for $n>1$, the number of vertices of degree 1 does not change if we attach a new vertex $v_{n}$ to a vertex with degree 1 and increases by 1 if we attach $v_{n}$ to vertices of degree larger than 1 after joining the vertex $v_{n}$. Assuming $n$ is multiple of $m$, that is, $n=k m$, where $k$ is integer number, and taking expectation of $D_{n}(1)$, we obtain

$$
\begin{aligned}
\operatorname{ED}_{k m}(1)= & \left(1-\frac{1}{2(k m-1)}\right) \operatorname{ED}_{k m-1}(1)+1 \\
= & \left(1-\frac{1}{2(k m-1)}\right) \\
& \times\left[\operatorname{ED}_{k m-2}(1)\left(1-\frac{1}{k m-2}\right)+1\right]+1 \\
= & \left(1-\frac{1}{2(k m-1)}\right)\left(1-\frac{1}{k m-2}\right) \operatorname{ED}_{k m-2}(1) \\
& +\left(1-\frac{1}{2(k m-1)}\right)+1 .
\end{aligned}
$$

The first equation shows that when we add a new vertex and link it to one of existing vertices with preferential attachment, the number of vertex increases by 1 , while the second equation comes from the uniform attachment. Continuing the iteration and noticing the boundary condition $\operatorname{ED}_{1}(1)=$ 0 , we have

$$
\begin{aligned}
\mathrm{ED}_{k m}(1)= & \sum_{j=1}^{k m} \prod_{i=0}^{[j / m]}\left(1-\frac{1}{2[(k-i) m-1]}\right) \\
& \times \prod_{v=2}^{j}\left(1-\frac{1}{k m-v}\right) \\
& \times\left(\prod_{s=0}^{[j / m]}\left(1-\frac{1}{(k-i) m-1}\right)\right)^{-1} .
\end{aligned}
$$

Considering the term

$$
\begin{aligned}
& \prod_{i=0}^{[j / m]}\left(1-\frac{1}{2[(k-i) m-1]}\right) \prod_{v=2}^{j}\left(1-\frac{1}{k m-v}\right) \\
& \quad \times\left(\prod_{s=0}^{[j / m]}\left(1-\frac{1}{(k-i) m-1}\right)\right)^{-1},
\end{aligned}
$$

we have

$$
\begin{aligned}
e^{\ln } & \prod_{i=0}^{[j / m]}(1-(1 / 2[(k-i) m-1])) \prod_{v=2}^{j}(1-(1 /(k m-v)))-\ln \prod_{s=0}^{[j / m]}(1-(1 /((k-i) m-1))) \\
& =e^{-\sum_{i=0}^{[j / m]}(1 / 2[(k-i) m-1])-\sum_{v=2}^{j}(1 /(k m-v))+\sum_{i=0}^{[j / m]}(1 /((k-i) m-1))} \\
& =e^{-(1 /(2 k-1)) \int_{t=0}^{[j / m]}(1 /(1-(m t /(2 k-1)))) d t-(1 / k m) \int_{v=2}^{j}(1 /(1-(v / k m))) d v+(1 /(k m-1)) \int_{t=0}^{[j / m]}(1 /(1-(m t /(k m-1)))) d t} \\
& =\left(1-\frac{j}{k m}\right)^{(1-(1 / 2 m))} .
\end{aligned}
$$

We obtain that

$$
\begin{aligned}
\operatorname{ED}_{k m}(1) & =\sum_{j=1}^{k m}\left(1-\frac{j}{k m}\right)^{(1-(1 / 2 m))} \\
& =\frac{2 m}{4 m-1} \cdot k m
\end{aligned}
$$

when $n$ is not a multiple of $m$, assuming $n=k^{\prime} m+s, 1<s<$ $m$, where $k^{\prime}$ is an integer number; we also obtain

$$
\begin{aligned}
& =\operatorname{ED}_{k^{\prime} m+s-1}(1)\left(1-\frac{1}{k^{\prime} m+s-1}\right)+1 \\
& =\cdots
\end{aligned}
$$$$
\operatorname{ED}_{k^{\prime} m+s}(1)
$$ 


$$
\begin{aligned}
= & \left(1-\frac{1}{k^{\prime} m+s-1}\right) \\
& \times\left(1-\frac{1}{k^{\prime} m+s-2}\right) \cdots\left(1-\frac{1}{k^{\prime} m}\right) \mathrm{ED}_{k^{\prime} m}^{m}(1) \\
& +\left(1-\frac{1}{k^{\prime} m+s-1}\right) \\
& \times\left(1-\frac{1}{k^{\prime} m+s-2}\right) \cdots\left(1-\frac{1}{k^{\prime} m+1}\right) \\
& +\cdots+\left(1-\frac{1}{k^{\prime} m+s-1}\right)\left(1-\frac{1}{k^{\prime} m+s-2}\right) \\
& +\left(1-\frac{1}{k^{\prime} m+s-1}\right) .
\end{aligned}
$$

When $n$ is large enough, we can see that

$$
\mathrm{ED}_{k^{\prime} m}(1)=\mathrm{ED}_{k^{\prime} m+s}(1)(1+o(1))
$$

As a result, we have

$$
\mathrm{ED}_{n}(1)=\frac{2 m}{4 m-1} n
$$

Now we discuss the number of degree 2 in the network; we have the following.

Lemma 2. For $n>2$,

$$
E D_{n}(2)=\frac{2 m-1}{8 m-2} n
$$

Proof. We prove the case that $n$ is a multiple of $m$ and assume $n=k m$, where $k$ is an integer number; considering the expectation of $D_{n}(2)$, we have

$$
\begin{aligned}
& \mathrm{ED}_{k m}(2)=\left(1-\frac{2}{2(k m-1)}\right) \mathrm{ED}_{k m-1}(2)+\frac{\mathrm{ED}_{k m-1}(1)}{2(k m-1)} \\
&=\left(1-\frac{1}{k m-1}\right)\left[\mathrm{ED}_{k m-2}(2)\left(1-\frac{1}{k m-2}\right)\right. \\
&\left.+\frac{\mathrm{ED}_{k m-2}(1)}{k m-2}\right]+\frac{\mathrm{ED}_{k m-1}(1)}{2(k m-1)} .
\end{aligned}
$$

Noticing the boundary condition $\operatorname{ED}_{2}(2)=0$ and Lemma 1 , we have

$$
\begin{aligned}
\operatorname{ED}_{k m}(2)= & \left(\sum_{j=1}^{k m} \prod_{i=1}^{j}\left(1-\frac{1}{k m-i}\right)\right. \\
& \left.-\frac{1}{2} \sum_{j=1}^{k} \prod_{v=1}^{j m}\left(1-\frac{1}{k m-v}\right)\right) \frac{2 m}{4 m-1} .
\end{aligned}
$$

By the estimation $\ln (1+x)=x$ and the fact that

$$
\begin{aligned}
& \prod_{i=1}^{j}\left(1-\frac{1}{k m-i}\right)=1-\frac{j}{k m}, \\
& \prod_{v=0}^{j m}\left(1-\frac{1}{k m-v}\right)=1-\frac{j}{k},
\end{aligned}
$$

we obtain that

$$
\begin{aligned}
\operatorname{ED}_{k m}(2) & =\frac{2 m}{4 m-1}\left[\sum_{j=1}^{k m}\left(1-\frac{j}{k m}\right)-\frac{1}{2} \sum_{j=0}^{k}\left(1-\frac{j}{k}\right)\right] \\
& =k m \frac{2 m}{4 m-1}\left[\int_{0}^{1}(1-x) d x-\frac{1}{2} \int_{0}^{1}(1-x) d x\right] \\
& =\frac{2 m-1}{8 m-2} \cdot k m .
\end{aligned}
$$

The case $n$, which is not a multiple of $m$, is the same as Lemma 1, just a little tedious.

\section{Asymptotic Degree Distribution of Network}

Let

$$
p_{k}(n)=\frac{D_{n}(k)}{n}
$$

denote the proportion of vertices with degree $k$ at time $n$. Considering the expectation of $D_{n}(k)$, we have the following theorem.

Theorem 3. For arbitrary $k>1$ and $n$, the expectation of the number of degree $k$ satisfies

$$
E D_{n}(k)=\frac{2 m}{4 m-1} \prod_{i=2}^{k} \frac{2 m+i-3}{4 m+j-2} n
$$

Proof. The case $k=1,2$ is just the result of Lemmas 1 and 2 . Assume the result is true for $k$; that is,

$$
\operatorname{ED}_{n}(k)=\frac{2 m}{4 m-1} \prod_{i=2}^{k} \frac{2 m+i-3}{4 m+j-2} n
$$


We will prove the result is true for $k+1$. We just prove the case $n$ is a multiple of $m$; that is, $n=l m$, where $l$ is integer number. From the network constructed, we have

$$
\begin{aligned}
\mathrm{ED}_{m l}(k+1) \\
=\left(1-\frac{k+1}{2(m l-1)}\right) \mathrm{ED}_{m l-1}(k+1) \\
+\frac{k}{2(m l-1)} \mathrm{ED}_{m l-1}(k) \\
=\left(1-\frac{k+1}{2(m l-1)}\right) \\
\quad \times\left[\mathrm{ED}_{m l-2}(k+1)\left(1-\frac{1}{m l-2}\right)+\frac{\mathrm{ED}_{m l-2}(k)}{m l-2}\right] \\
+\frac{k}{2(m l-1)} \mathrm{ED}_{m l-1}(k) .
\end{aligned}
$$

Continuing the iteration and noticing the boundary condition $\mathrm{ED}_{s}(k+1)=0, s<k$, we obtain that

$$
\begin{aligned}
\mathrm{ED}_{m l}(k+1) & \frac{2 m}{4 m-1} \prod_{i=2}^{k} \frac{2 m+i-3}{4 m+j-2} \\
& \times \sum_{j=1}^{l m} \prod_{i=0}^{[j / m]}\left(1-\frac{k+1}{2(l-i) m-1}\right) \prod_{v=1}^{j}\left(1-\frac{1}{l m-v}\right) \\
& \times\left(\prod_{i=0}^{[j / m]}\left(1-\frac{1}{(l-i) m-1}\right)\right)^{-1}+\frac{k-2}{2} \frac{2 m}{4 m-1} \\
& \times \prod_{i=2}^{k} \frac{2 m+i-3}{4 m+j-2} \sum_{j=1}^{l} \prod_{i=0}^{j}\left(1-\frac{k+1}{2(l-i) m-1}\right) \\
& \times \prod_{v=1}^{j}\left(1-\frac{1}{l m-v}\right) \times\left(\prod_{i=0}^{j}\left(1-\frac{1}{(l-i) m-1}\right)\right)^{-1} .
\end{aligned}
$$

Noticing the fact that

$$
\begin{aligned}
& \prod_{i=0}^{[j / m]}\left(1-\frac{k+1}{2(l-i) m-1}\right) \prod_{v=1}^{j}\left(1-\frac{1}{l m-v}\right) \\
& \quad \times\left(\prod_{i=0}^{[j / m]}\left(1-\frac{1}{(l-i) m-1}\right)\right)^{-1} \\
& =\left(1-\frac{j}{l m}\right)^{(((k-1) / 2 m)+1)},
\end{aligned}
$$

$$
\begin{aligned}
& \prod_{i=0}^{j}\left(1-\frac{k+1}{2(l-i) m-1}\right) \prod_{v=1}^{j m}\left(1-\frac{1}{l m-v}\right) \\
& \quad \times\left(\prod_{i=0}^{j}\left(1-\frac{1}{(l-i) m-1}\right)\left(1-\frac{j}{l m}\right)^{(((k-1) / 2 m)+1)}\right)^{-1} \\
& =\left(1-\frac{j}{l}\right)^{(((k-1) / 2 m)+1)} .
\end{aligned}
$$

We obtain

$$
\begin{aligned}
& \mathrm{ED}_{l m}(k+1) \\
& =\frac{2 m}{4 m-1} \prod_{i=2}^{k} \frac{2 m+i-3}{4 m+j-2} \\
& \times \sum_{j=1}^{l m}\left(1-\frac{j}{l m}\right)^{(((k-1) / 2 m)+1)}+\frac{k-2}{2} \frac{2 m}{4 m-1} \\
& \times \prod_{i=2}^{k} \frac{2 m+i-3}{4 m+j-2} \sum_{j=1}^{l}\left(1-\frac{j}{l}\right)^{(((k-1) / 2 m)+1)} \\
& =\frac{2 m}{4 m-1} \prod_{i=2}^{k} \frac{2 m+i-3}{4 m+j-2} \int_{0}^{1}(1-x)^{(((k-1) / 2 m)+1)} d x \cdot \operatorname{lm} \\
& +\frac{k-2}{2} \frac{2 m}{4 m-1} \\
& \times \prod_{i=2}^{k} \frac{2 m+i-3}{4 m+j-2} \int_{0}^{1}(1-x)^{(((k-1) / 2 m)+1)} d x \cdot l \\
& =\frac{2 m}{4 m-1} \prod_{i=2}^{k} \frac{2 m+i-3}{4 m+j-2}\left(1+\frac{k-2}{2 m}\right) \\
& \cdot \frac{2 m}{4 m+k-1} \cdot \operatorname{lm} \\
& =\frac{2 m}{4 m-1} \prod_{i=2}^{k+1} \frac{2 m+i-3}{4 m+j-2} \cdot \operatorname{lm} \text {. }
\end{aligned}
$$

The result is true for $k+1$.

From Theorem 3, we can see that $\lim _{n \rightarrow \infty}\left(\operatorname{ED}_{n}(k) / n\right)$ exists; we denote it by $p_{k}$. Now we consider the relation of $p_{k}$ and $p_{k}(n)$; we introduce the following lemma.

Lemma 4. There exists a bound constant $C(k)$ such that for arbitrary $a>0$,

$$
P\left(\left|D_{n}(k)-E D_{n}(k)\right| \geq a\right) \leq 2 e^{-a^{2} / 2 C(k)^{2} n} .
$$

Proof. Let $\mathfrak{F}_{n}=\sigma\left(D_{1}(1), \ldots, D_{k}(1), D_{k}(2), \ldots D_{k}(k), \ldots\right.$, $\left.D_{n}(1), \ldots D_{n}(k) \ldots, D_{n}(n)\right)$ denote the $\sigma$-algbra. For $m=$ $0,1, \ldots, n$, we define

$$
M_{m}=E\left(D_{k}(n) \mid \mathfrak{F}_{m}\right) .
$$


By the tower property of conditional expectation and the fact that the $\sigma$-algbra $\mathfrak{F}_{n}$ can be deduced from $\mathfrak{F}_{n+1}$, we obtain that, for $m<n$,

$$
\begin{aligned}
E\left(M_{m+1} \mid \mathfrak{F}_{m}\right) & =E\left[E\left(D_{n}(k) \mid \mathfrak{F}_{m+1}\right) \mid \mathfrak{F}_{m}\right] \\
& =E\left(D_{n}(k) \mid \mathfrak{F}_{m}\right) \\
& =M_{m} .
\end{aligned}
$$

Noticing the fact that

$$
E\left[\left|M_{m}\right|\right]=E M_{m}=\operatorname{ED}_{n}(k)<n<\infty
$$

we have $\left\{M_{m}\right\}_{m=0}^{n}$ as a martingale sequence. According to the definition of the $\sigma$-algbra, we know the $\mathfrak{F}_{0}$ has no information of the network and $\mathfrak{F}_{n}$ has the whole information, so we have

$$
\begin{gathered}
M_{0}=E\left[D_{n}(k) \mid \mathfrak{F}_{0}\right]=\operatorname{ED}_{n}(k), \\
M_{n}=E\left[D_{n}(k) \mid \mathfrak{\mho}_{n}\right]=D_{n}(k) .
\end{gathered}
$$

Therefore, we have

$$
D_{n}(k)-\mathrm{ED}_{n}(k)=M_{n}-M_{0}=\sum_{j=0}^{n}\left(M_{j+1}-M_{j}\right) .
$$

Now we prove that there exists a bound constant $C(k)$, such that $\left|M_{j+1}-M_{j}\right| \leq C(k)$. We will prove the result by induction. For the case $k=1$, we have

$$
\begin{aligned}
& \left|M_{j+1}-M_{j}\right| \\
& =\left|E\left(D_{n}(1) \mid \mathfrak{F}_{j+1}\right)-E\left(D_{n}(1) \mid \mathfrak{F}_{j}\right)\right| \\
& =\mid E\left(D_{n}(1)-D_{n-1}(1) \mid \mathfrak{\mho}_{j+1}\right) \\
& -E\left(D_{n}(1)-D_{n-1}(1) \mid \mathfrak{F}_{j}\right) \\
& +E\left(D_{n-1}(1) \mid \mathfrak{\Im}_{j+1}\right)-E\left(D_{n-1}(1) \mid \mathfrak{F}_{j}\right) \mid \\
& =\mid E\left(E\left(D_{n}(1)-D_{n-1}(1) \mid \mathfrak{F}_{n-1}\right) \mid \mathfrak{F}_{j+1}\right) \\
& -E\left(E\left(D_{n}(1)-D_{n-1}(1) \mid \mathfrak{F}_{n-1}\right) \mid \mathfrak{F}_{j}\right) \\
& +E\left(D_{n-1}(1) \mid \mathfrak{F}_{j+1}\right)-E\left(D_{n-1}(1) \mid \mathfrak{F}_{j}\right) \mid \\
& =\left(1-\frac{1}{n-1}\right) \\
& \times\left|E\left(D_{n-1}(1) \mid \mathfrak{F}_{j+1}\right)-E\left(D_{n-1}(1) \mid \mathfrak{F}_{j}\right)\right| .
\end{aligned}
$$

Continuing the iteration and noticing the fact that $E\left(D_{m}(1) \mid \mathfrak{F}_{j+1}\right)-E\left(D_{m}(1) \mid \mathfrak{F}_{j}\right)=0$, for $m<j$, we obtain that

$$
\begin{aligned}
\left|M_{j+1}-M_{j}\right| & \prod_{i=j}^{n-1}\left(1-\frac{1}{i}\right) \prod_{s=1}^{[n / m]}\left(1-\frac{1}{2(s m-1)}\right) \\
& \times\left(\prod_{s=1}^{[n / m]}\left(1-\frac{1}{s m-1}\right)\right)^{-1} \\
& \cdot\left|E\left(D_{j+1}(1) \mid \mathfrak{\mho}_{j+1}\right)-E\left(D_{j+1}(1) \mid \mathfrak{\mho}_{j}\right)\right| \\
= & \prod_{i=j}^{n-1}\left(1-\frac{1}{i}\right) \prod_{s=1}^{[n / m]}\left(1-\frac{1}{2(s m-1)}\right) \\
& \times\left(\prod_{s=1}^{[n / m]}\left(1-\frac{1}{s m-1}\right)\right)^{-1} \\
& \cdot\left|\left(D_{j+1}(1)-D_{j}(1)\right)-E\left(D_{j+1}(1)-D_{j}(1) \mid \mathfrak{F}_{j}\right)\right| .
\end{aligned}
$$

Obviously,

$$
\begin{gathered}
\left|D_{j+1}(1)-D_{j}(1)\right| \leq 1, \\
\prod_{i=j}^{n-1}\left(1-\frac{1}{i}\right) \prod_{s=1}^{[n / m]}\left(1-\frac{1}{2(s m-1)}\right) \\
\times\left(\prod_{s=1}^{[n / m]}\left(1-\frac{1}{s m-1}\right)\right)^{-1} \leq 1,
\end{gathered}
$$

so we have

$$
\left|M_{j+1}-M_{j}\right| \leq 2
$$

Assume the result is true for $k$; that is, there exists a bound constant $C(k)$, such that

$$
\left|M_{j+1}-M_{j}\right| \leq C(k) .
$$

For $k+1$, by the definition of $M_{j+1}$, we have

$$
\begin{aligned}
& \left|M_{j+1}-M_{j}\right| \\
& =\left|E\left(D_{n}(k+1) \mid \mathfrak{F}_{j+1}\right)-E\left(D_{n}(k+1) \mid \mathfrak{F}_{j}\right)\right| \\
& =\left(1-\frac{1}{n-1}\right) \\
& \quad \times\left|E\left(D_{n}(k+1) \mid \mathfrak{\Im}_{j+1}\right)-E\left(D_{n-1}(k+1) \mid \mathfrak{F}_{j}\right)\right| \\
& \quad+\frac{1}{n-1}\left[E\left(D_{n-1}(k) \mid \mathfrak{F}_{j+1}\right)-E\left(D_{n-1}(k) \mid \mathfrak{F}_{j}\right)\right] .
\end{aligned}
$$


Continuing the iteration and using the assumption for $k$, we obtain that

$$
\begin{aligned}
& \left|M_{j+1}-M_{j}\right| \\
& \leq \prod_{v=j+1}^{n-1}\left(1-\frac{1}{v}\right) \prod_{i=[(j+1) / m]}^{[n / m]}\left(1-\frac{k+1}{2(i m-1)}\right) \\
& \times\left(\prod_{i=[(j+1) / m]}^{[n / m]}\left(1-\frac{1}{(i m-1)}\right)\right)^{-1} \\
& \cdot\left|E\left(D_{j+1}(k+1) \mid \mathfrak{F}_{j+1}\right)-E\left(D_{j+1}(k+1) \mid \mathfrak{F}_{j}\right)\right| \\
& +\sum_{v=1}^{n-j-1} \prod_{s=1}^{v}\left(1-\frac{1}{n-s}\right) \frac{1}{n-v-1} C(k) \\
& +\sum_{v=0}^{[(n-j-1) / m]} \prod_{s=1}^{n-([n / m]-v) m}\left(1-\frac{1}{n-s}\right) \\
& \cdot \prod_{s=0}^{v}\left(1-\frac{k+1}{2(([n / m]-s) m-1)}\right) \\
& \times\left(\prod_{s=0}^{j}\left(1-\frac{1}{([n / m]-s) m-1} C(k)\right)\right)^{-1} .
\end{aligned}
$$

Noticing the fact that $1-((k+1) / 2 j)<1-(1 / j)$ and

$$
\begin{gathered}
\prod_{v=j+1}^{n-1}\left(1-\frac{1}{v}\right) \prod_{i=[(j+1) / m]}^{[n / m]}\left(1-\frac{k+1}{2(i m-1)}\right) \\
\times\left(\prod_{i=[(j+1) / m]}^{[n / m]}\left(1-\frac{1}{(i m-1)}\right)\right)^{-1} \leq 1,
\end{gathered}
$$

we obtain that

$$
\begin{aligned}
\left|M_{j+1}-M_{j}\right| & \leq 2+\frac{n-j-1}{n-1} C(k)+\frac{[(n-j-1) / m]}{n-1} C(k) \\
& \leq 2+C(k)\left(1+\frac{1}{m}\right) .
\end{aligned}
$$

We just let $C(k+1)=2+C(k)(1+(1 / m))$ and the result for $k+1$ is proved. By Asume-Hoeffding's inequality, we have the following for arbitrary $a>0$ :

$$
P\left(\left|D_{n}(k)-\mathrm{ED}_{n}(k)\right| \geq a\right) \leq 2 e^{-a^{2} / 2 C(k)^{2} n} .
$$

Theorem 5. For a fixed $k$, one has

$$
\lim _{n \rightarrow \infty} p_{k}(n)=p_{k} \quad \text { a.e. }
$$

Proof. By the Borel-Cantelli Lemma, we need to prove the following for arbitrary $\varepsilon$ :

$$
\sum_{n=1}^{\infty} P\left(\left|p_{k}(n)-p_{k}\right|>\varepsilon\right)<\infty .
$$

We have

$$
\begin{aligned}
\sum_{n=1}^{\infty} P & \left(\left|p_{k}(n)-p_{k}\right|>\varepsilon\right) \\
= & \sum_{n=1}^{\infty} P\left(\left|\frac{D_{n}(k)}{n}-\frac{\mathrm{ED}_{n}(k)}{n}+\frac{\mathrm{ED}_{n}(k)}{n}-p_{k}\right|>\varepsilon\right) \\
\leq & \sum_{n=1}^{\infty} P\left(\left|\frac{D_{n}(k)}{n}-\frac{\mathrm{ED}_{n}(k)}{n}\right| \geq \frac{\varepsilon}{2}\right) \\
& +\sum_{n=1}^{\infty} P\left(\left|\frac{\mathrm{ED}_{n}(k)}{n}-p_{k}\right| \geq \frac{\varepsilon}{2}\right) .
\end{aligned}
$$

Noticing that $\lim _{n \rightarrow \infty} \operatorname{ED}_{k}(n) / n=p_{k}$ and using Lemma 4, we obtain that there exists $N$, such that

$$
\begin{aligned}
& \sum_{n=1}^{\infty} P\left(\left|p_{k}(n)-p_{k}\right|>\varepsilon\right) \\
& \quad \leq \sum_{n=1}^{\infty} P\left(\left|D_{n}(k)-\mathrm{ED}_{n}(k)\right| \geq \frac{\varepsilon}{2} n\right)+N \\
& \quad \leq \sum_{n=1}^{\infty} 2 e^{-\left(\varepsilon^{2} / 4\right) n}+N \\
& \quad<\infty .
\end{aligned}
$$

Remark 6. As a result, we can see that the distribution $p_{k}$ obeys the following rule.

When $m \ll k, p_{k} \propto k^{-(2 m+1)}$, the degree distribution obeys power law; when $m \gg k, p_{k} \propto 2^{-k}$, the degree distribution obeys exponential distribution; otherwise, $p_{k}=$ $(2 m /(4 m-1)) \prod_{j=2}^{k}((2 m+j-3) /(4 m+j-2))$.

\section{Conflict of Interests}

The authors declare that there is no conflict of interests.

\section{Acknowledgments}

Zhimin Li was partially supported by National Natural Science Foundation of China (71171003 and 71271003), Anhui Natural Science Foundation (nos. 10040606Q03 and 1208085QA04), and Key University Science Research Project of Anhui Province (KJ2013A044).

\section{References}

[1] F. Chung and L. Y. Lu, Complex Graphs and Networks, American Mathematical Society, 2006.

[2] X. Zhang and L. Yang, "A fiber Bragg grating quasi-distributed sensing network with a wavelength-tunable chaotic fiber laser," Systems Science and Control Engineering, vol. 2, no. 1, pp. 268274, 2014.

[3] H. Ahmada and T. Namerikawa, "Extended Kalman filter-based mobile robot localization with intermittent measurements," 
Systems Science and Control Engineering, vol. 1, no. 1, pp. 113126, 2013.

[4] D. Ding, Z. Wang, H. Dong, and H. Shu, "Distributed Ho state estimation with stochastic parameters and nonlinearities through sensor networks: the finite-horizon case," Automatica, vol. 48, no. 8, pp. 1575-1585, 2012.

[5] D. Ding, Z. Wang, J. Hu, and H. Shu, "Dissipative control for state-saturated discrete time-varying systems with randomly occurring nonlinearities and missing measurements," International Journal of Control, vol. 86, no. 4, pp. 674-688, 2013.

[6] Z. Wang, H. Dong, B. Shen, and H. Gao, "Finite-horizon $\mathrm{H}_{\infty}$ filtering with missing measurements and quantization effects," IEEE Transactions on Automatic Control, vol. 58, no. 7, pp. 17071718, 2013.

[7] H. Ahmada and T. Namerikawa, "Extended Kalman filter-based mobile robot localization with intermittent measurements," Systems Science \& Control Engineering, vol. 1, no. 1, pp. 113-126, 2013.

[8] G. Kumar and K. Kumar, "Network security-an updated perspective," Systems Science \& Control Engineering, vol. 2, no. 1, pp. 325-334, 2014.

[9] B. Bollobás, Random Graphs, Combrige University Press, Cambrige, UK, 2001.

[10] A. L. Barabási and R. Albert, "Emergence of scaling in random networks," Science, vol. 286, no. 5439, pp. 509-512, 1999.

[11] A. L. Barabási, R. Albert, and H. Jeong, "Mean-field theory for scale-free random networks," Physica A: Statistical Mechanics and its Applications, vol. 272, no. 1, pp. 173-187, 1999.

[12] B. Bollobás, O. Riordan, J. Spencer, and G. Tusnády, "The degree sequence of a scale-free random graph process," Random Structures \& Algorithms, vol. 18, no. 3, pp. 279-290, 2001.

[13] S. N. Dorogovtsev, J. F. F. Mendes, and A. N. Samukhin, "Structure of growing networks with preferential linking," Physical Review Letters, vol. 85, no. 21, pp. 4633-4636, 2000.

[14] E. Drine, M. Enachescu, and M. Mitzenmacher, "Variations on random graph models for the web," Tech. Rep., Havard University, Deparment of Computer Science, 2001.

[15] P. L. Krapivsky and S. Redner, "Organization of growing random networks," Physical Review E-Statistical, Nonlinear, and Soft Matter Physics, vol. 63, no. 6, Article ID 066123, 2001.

[16] Z. Li, "Branching structure and maximum degree of an evolving random tree," Theoretical and Mathematical Physics, vol. 166, no. 2, pp. 270-277, 2011. 


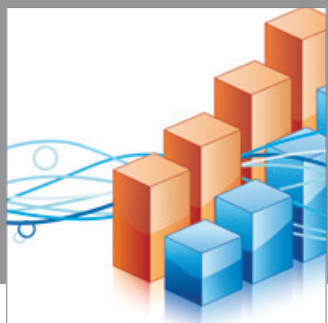

Advances in

Operations Research

mansans

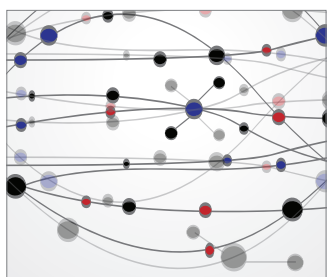

The Scientific World Journal
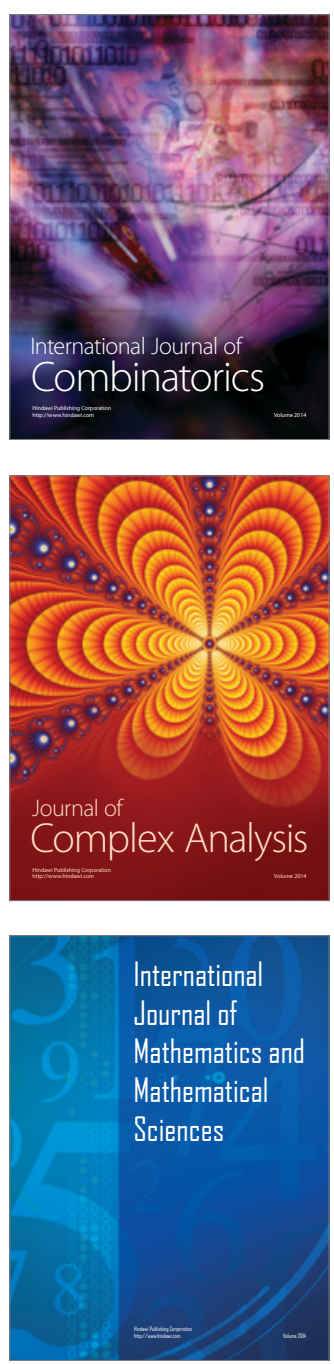
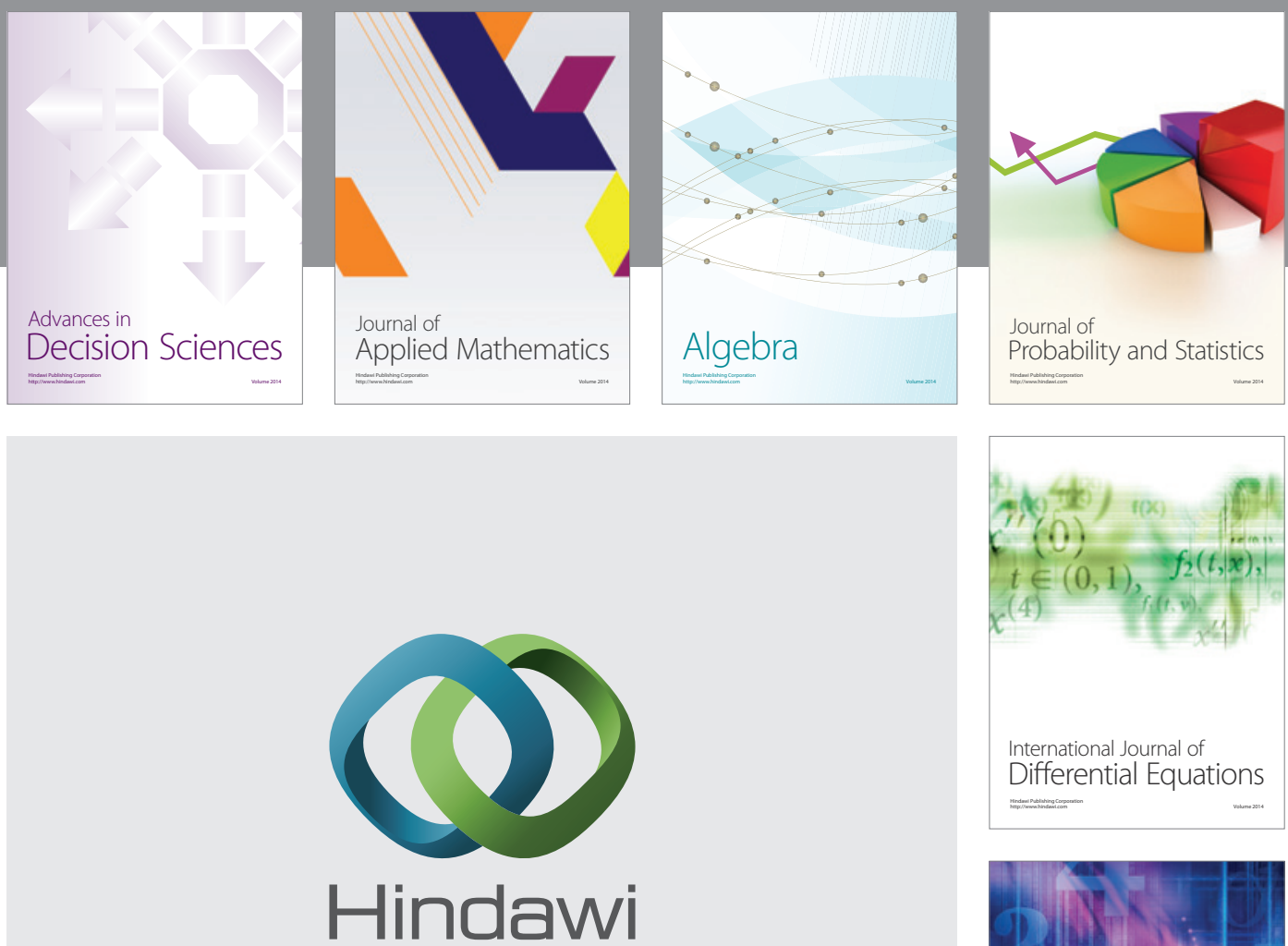

Submit your manuscripts at http://www.hindawi.com
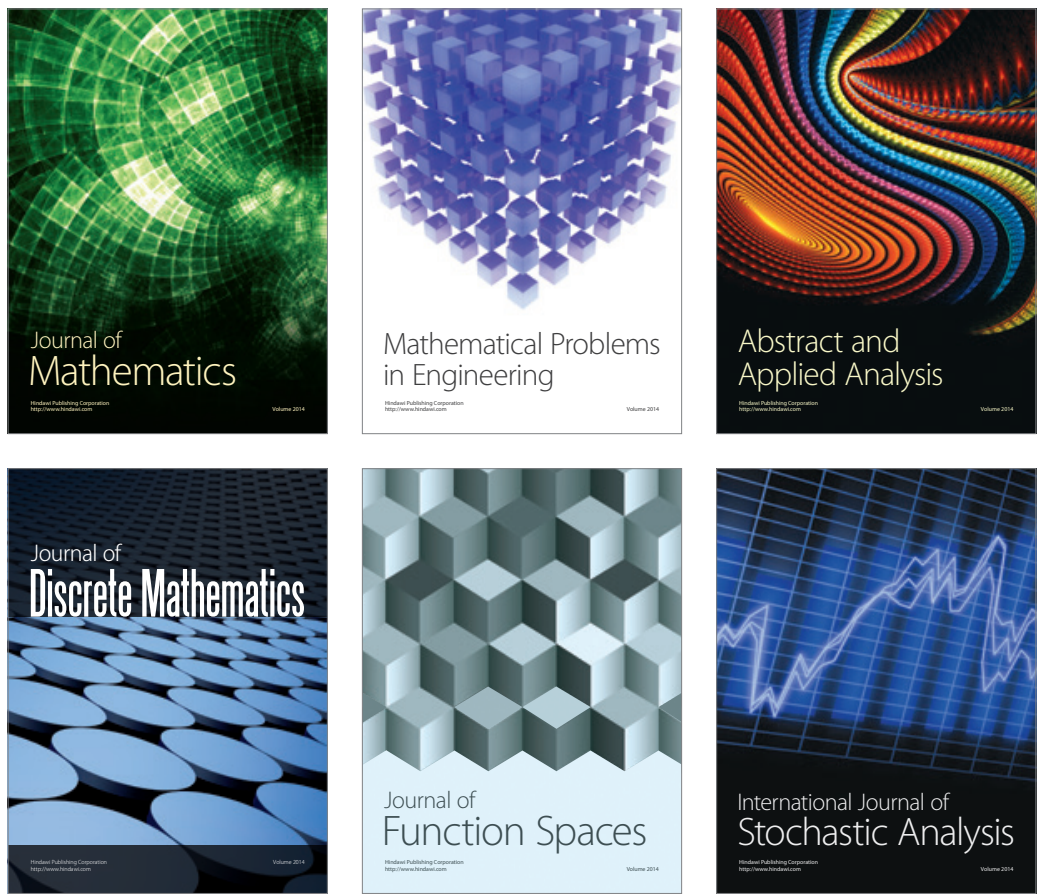

Journal of

Function Spaces

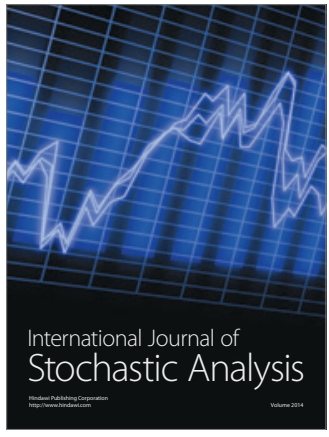

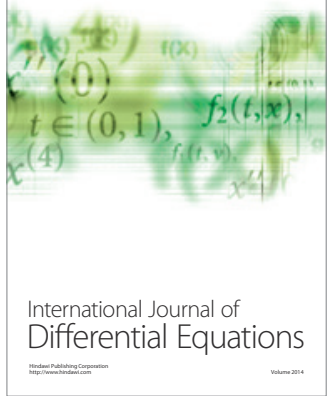
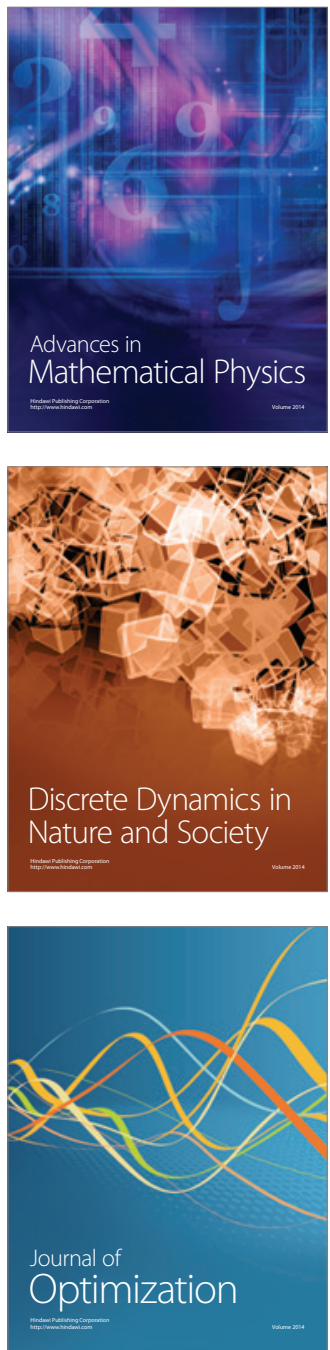